\title{
IMUNIZAÇÃO EM CRIANÇA EXPOSTA OU INFECTADA PELO HIV EM UM SERVIÇO DE IMUNOBIOLÓGICOS ESPECIAIS
}

\author{
Immunization in children exposed or infected by hiv in a service of special \\ immunobiologicals \\ Inmunización en niño expuesto o infectado por el vih en un servicio de inmunobiológicos \\ especiales
}

\author{
Maria do Socorro Mendonça Sherlock \\ Ana Luíza Paula de Aguiar Lélis
}

\author{
Maria Vera Lúcia Moreira Leitão Cardoso² \\ Natália Rodrigues Oliveira ${ }^{5}$
}

Márcia Maria Coelho Oliveira Lopes ${ }^{3}$

\section{RESUMO}

Objetivou-se analisar a situação vacinal de crianças expostas ou infectadas pelo HIV atendidas no programa de imunobiológicos especiais (CRIES). Estudo documental, retrospectivo, desenvolvido no CRIES de um hospital público pediátrico, em Fortaleza-CE. A amostra consistiu em 125 prontuários de crianças atendidas no período de janeiro de 2006 a setembro de 2009. Conforme 0 Ministério da Saúde, percebeu-se que a maioria das crianças iniciou a imunização aos 2 meses de idade, destas 65 (52,0\%) com o esquema vacinal injetável contra pólio e $68(64,8 \%)$ contra pneumococo, mas apresentaram redução da demanda no seguimento do calendário ao avançar a idade. Assim, a baixa adesão ao calendário de vacina especial foi perceptível, o que implica rompimento do processo imunológico das crianças expostas, tornando-se imprescindível um trabalho educativo pelos profissionais de saúde e a conscientização da população, visando à prevenção de doenças evitáveis no grupo de risco estudado.

Palavras-chave: Saúde da Criança. HIV. Imunossupressão. Imunização. Enfermagem Pediátrica.

\begin{abstract}
It was aimed to analyze the vaccination status of children exposed or infected by HIV assisted in the program of special immunobiological (CRIES). Documentary retrospective study developed in the CRIES of a public pediatric hospital in FortalezaCE. The sample consisted of 125 registers of children treated from January 2006 to September 2009. It was verified that as the Ministry of Health indicates, most children started immunization at 2 months of age, 65 (52.0\%) of those with the injectable polio vaccine schedule and $68(64.8 \%)$ against pneumococcus, but presented reduction on the demand in following the schedule along the years. Thus, the poor adherence to the schedule of the special vaccine was noticeable, which implicates in the breaking of the process of immune children exposed, becoming a vital educational work by health professionals and the public awareness aiming the prevention of preventable diseases in the risk group studied.
\end{abstract}

Keywords: Child Health. HIV. Immunosuppression. Immunization. Pediatric Nursing.

\section{Resumen}

El objetivo fue analizar la situación relativa a las vacunas de niños expuestos o infectados por el VIH atendidos en el programa de inmunobiológicos especiales (CRIES). Estudio documental, retrospectivo, desarrollado en un hospital público pediátrico, en Fortaleza-CE. La muestra fue de 125 registros médicos de niños atendidos de enero de 2006 a septiembre de 2009. Se percibió que según el Ministerio de la Salud indica, la mayoría de los niños comenzó la inmunización a los 2 meses de edad, de estos 65 $(52,0 \%)$ con el calendario de vacunas inyectable contra la poliomielitis y $68(64,8 \%)$ contra el neumococo, pero presentaron reducción de la demanda en el seguimiento del calendario al avanzar la edad. Por lo tanto, la baja adherencia al calendario de vacunación especial fue notable, lo que implica la ruptura del proceso inmunológico de los niños expuestos, convirtiéndose en una acción educativa por los profesionales de salud y la conciencia de la población, para la prevención de las enfermedades prevenibles en el grupo de riesgo estudiado.

Palabras clave: Salud del Niño. VIH. Inmunosupresión. Inmunización. Enfermería Pediátrica.

\footnotetext{
'Enfermeira. Mestre em Enfermagem em Saúde Comunitária/UFC. Coordenadora do Curso de Especialização em Enfermagem Neonatal/UFC. Membro do Projeto Saúde do Binômio Mãe-filho/SABIMF/UFC.Fortaleza-Ce, Brasil. E-mail:socorrosherlock@yahoo.com.br;'Enfermeira. Pós-Doutorado Canadá. Profa. Associada do Departamento de Enfermagem - FFOE/UFC. Pesquisador CNPq. Coordenadora do Projeto Saúde do Binômio Mãe-filho/SABIMF/UFC. Fortaleza-Ce, Brasil. E-mail: cardoso@ufc.br ;'Enfermeira da Unidade Neonatal da MEAC/UFC. Membro do Projeto Saúde do Binômio Mãe-filho/SABIMF/ UFC Doutoranda da Pós-Graduação da FFOE/UFC. Fortaleza-Ce, Brasil. E-mail: enf.marcya@gmail.com; ${ }^{4}$ Enfermeira. Mestranda pelo Programa de Pósgraduação em Enfermagem da Universidade Federal do Ceará (UFC). Bolsista CAPES. Membro do Projeto Saúde do Binômio Mãe-filho/SABIMF/UFC. Av. Filomeno Gomes, n⿳0100. Jacarecanga. CEP: 60010280 - Fortaleza- CE, Brasil. Email: aninhanurse@hotmail.com; ${ }^{5}$ Acadêmica de Enfermagem da UFC. Bolsista PIBIC/CNPq. Membro do Projeto Saúde do Binômio Mãe-filho/SABIMF/UFC. Fortaleza-CE, Brasil. Email: natalia87_r@yahoo.com.br
} 


\section{INTRODUÇÃO}

0 Programa Nacional de Imunizações (PNI), sob responsabilidade do Ministério da Saúde, contribui sobremodo para a mudança do perfil epidemiológico de doenças imunopreviníveis, cujos resultados são considerados uma das maiores conquistas da saúde pública do Brasil.

Com a Síndrome da Imunodeficiência Adquirida (AIDS), até junho de 2008, notificaram-se ao Ministério da Saúde 17.304 casos, em menores de 13 anos de idade, 11.607 por transmissão vertical. ${ }^{1-2}$ Estima-se que em $65 \%$ dos casos, a transmissão ocorra durante o trabalho de parto e parto; $35 \%$, intraútero, principalmente nas últimas semanas de gestação, $\mathrm{e}$ também pelo aleitamento materno, ${ }^{3}$ cujos riscos adicionais de transmissão situam-se entre $7 \%$ e $22 \%$ e que se renovam a cada exposição da criança ao peito. A taxa de transmissão vertical do Vírus da Imunodeficiência Humana (HIV) alcança 25,5\%, ou seja, a cada 100 crianças nascidas de mães infectadas, 25 tornam-se expostas ao HIV. ${ }^{4}$

Entretanto, com os produtos imunobiológicos de moderna tecnologia e alto custo, instituídos pelo Ministério da Saúde, a transmissão reduziu-se a menos de 1\%, com as seguintes medidas preventivas: oferecimento rotineiro do teste sorológico para HIV no pré-natal e, em caso positivo, uso de drogas antirretrovirais na gestação, parto (cesariana), lquego-Zidov Udina (AZT), xarope ao recémnascido (RN) e desaconselhamento do aleitamento materno, substituindo-o por leite artificial. ${ }^{5-6}$

Além disso, destaca-se como aspecto importante o acompanhamento do RN exposto ao HIV, por isso recomendase aos profissionais o encaminhamento à consulta agendada em serviço especializado, após alta hospitalar da maternidade, visando ao acompanhamento clínico e laboratorial da criança exposta ao HIV, que se estende dos dois primeiros anos de vida, nos primeiros seis meses. A consulta é mensal e, no mínimo, trimestral a partir do $2^{\circ}$ semestre de vida.?

Apesar de medidas implementadas e disponíveis à população de gestantes infectadas pelo HIV, a cobertura insuficiente de mulheres testadas no pré-natal, principalmente em populações mais vulneráveis ao HIV, e a qualidade do pré-natal e da assistência ao parto estão ainda aquém do desejável. ${ }^{5}$ Apesar das dificuldades, a incidência de casos de AIDS em crianças vem decrescendo progressivamente no País. ${ }^{1,8}$

0 Ministério da Saúde, consciente da prevalência do HIV em gestantes e crianças expostas, bem como da suscetibilidade a determinadas infecções da população em geral, estabelece ações preventivas de resguardo do grupo do HIV. A esse respeito, em 1993, implantaram-se os Centros de Referência para Imunobiológicos Especiais (CRIES) que disponibilizam vacinas e imunobiológicos indicados para situações especiais, não disponíveis habitualmente em postos de saúde. ${ }^{9}$ Deve-se salientar que a criança não vacinada, além de manter-se suscetível à doença, representa risco para a população.

A imunização é o procedimento de melhor custo/ efetividade, com o objetivo de promoção e proteção do indivíduo. A vacinação configura-se defesa adquirida que, acrescida à defesa natural, evita a contaminação por algumas doenças infectocontagiosas na população com maior vulnerabilidade entre estudantes e profissionais de saúde. ${ }^{10}$

A prática assistencial de enfermagem e as evidências científicas sinalizam para o fato de que a atenção à saúde da criança avança em aspectos epidemiológicos, imunológicos, tecnológicos e socioeconômicos. Entretanto, considera-se crítica a situação de algumas crianças que nasceram com saúde comprometida e que, posteriormente, necessitaram de acompanhamento especializado, no intuito de promover a recuperação, com base no tratamento e na prevenção de doenças.

Diante da criança com suspeita clínica ou diagnóstico definitivo de HIV, questionou-se: quais os imunobiológicos da clientela no hospital em estudo? Como são os protocolos de encaminhamento de criança HIV para o CRIES? Quais as rotinas de enfermagem no serviço?

Diante dos questionamentos, surgiu o interesse pelo estudo, na busca por conhecimentos sobre a temática, bem como por fornecimento de informações atualizadas à equipe que presta diretamente assistência às crianças em situação de risco, em especial a que atende em CRIES. Ademais, pretendeu-se dos profissionais de enfermagem melhor compreensão acerca dos aspectos envolvidos em cuidados essenciais quanto de tecnologias imunológicas, que necessitam olactente exposto ou portador de HIV.

Nesse contexto, objetivou-se analisar a situação vacinal de crianças expostas ao HIV atendidas em um programa de imunobiológicos especiais.

\section{MÉTODO}

Tratou-se de estudo documental, retrospectivo, realizado em Hospital Infantil de grande porte, vinculado à rede pública estadual de saúde, conveniado com o Sistema Único de Saúde (SUS), em Fortaleza-CE-Brasil.

0 cenário foi o CRIES destinado ao atendimento de indivíduos portadores de quadros clínicos especiais, ou seja, com suscetibilidade aumentada às doenças ou ao risco de complicações para si ou para outros, decorrente de motivos biológicos como imunodepressão, asplenia, transplante, AIDS, por intolerância aos imunobiológicos comuns, devido a eventos adversos graves depois de recebê-los, por exposição inadvertida a agentes infecciosos, por motivos profissionais ou violência contra a pessoa. ${ }^{8}$

Nesse contexto, destaca-se que os profissionais da área de saúde tornam-se responsáveis pelo encaminhamento de crianças portadoras de quadros clínicos especiais para as unidades especializadas que dispõem das condições necessárias para os procedimentos, em conformidade com o Ministério da Saúde. 0 CRIES atende ao público que necessita de imunobiológicos especiais, de alta tecnologia e de altíssimo custo, de forma 
personalizada. Para uso da medicação, é necessário que a pessoa apresente prescrição com indicação médica e um relatório clínico sobre seu caso, bem como dos exames laboratoriais que a justifiquem. Os CRIES estão subordinados administrativamente às instituições nos quais estão implantados e tecnicamente às respectivas Secretarias de Saúde e a Coordenação do PNI. ${ }^{7}$

A equipe técnica do CRIES estudado era composta por um médico, duas enfermeiras e duas auxiliares de enfermagem, capacitados para atuar em imunizações. Às enfermeiras e ao médico cabiam o planejamento, a organização, a execução e a supervisão da dinâmica do serviço. Além disso, as enfermeiras realizavam triagem, recepção e atendimento à clientela, supervisão e conservação dos imunobiológicos. 0 CRIES funcionava em período integral para dispensação dos imunobiológicos em casos de urgência, inclusive nos períodos noturnos, finais de semana e feriados.

Quanto à estrutura física, era composto por duas salas para recepção, atendimento aos clientes e acondicionamento dos prontuários e informatização. Os procedimentos da sala de vacinação eram registrados em impressos padronizados que permitiam o conhecimento e acompanhamento do esquema de vacinação de cada criança, possibilitando que as informações fossem encaminhadas às instâncias estaduais e federais e contribuíssem para a implantação de estratégias de avaliação e melhoria no atendimento do serviço para a população.

A população constituiu-se de $4.094(100 \%)$ dos prontuários de crianças atendidas no CRIES, de janeiro de 2006 a setembro de 2009. Destes, foram escolhidos 125 (3\%) prontuários, que configuraram o tamanho da amostra e corresponderam a todas as crianças filhas de mães portadoras de HIV, critério de inclusão na amostra. Ressalta-se que alguns dados foram extraídos do livro registro, dos cadastros, dos formulários de encaminhamento e do controle de administração dos imunobiológicos especiais para complementar as informaç̃̃es dos prontuários, quando se fez necessário.

Para coleta de dados, elaborou-se formulário com as variáveis: data de nascimento, idade na vacinação, tipo de vacina, número de doses, procedência e instituição de origem do encaminhamento da criança, cujos nomes foram mantidos em sigilo e substituídos por letras como, por exemplo, Hospital A, Hospital B. Posteriormente, a análise dos dados foi realizada por meio do Programa Excell, utilizando-se da frequência simples e absoluta, sendo apresentados em gráfico e tabelas e respaldados pela literatura pertinente ao tema, embora se tenha percebido escassez de literatura específica sobre o tema, principalmente na Enfermagem.

Em concordância com a Resolução 196/96, do Conselho Nacional de Saúde, do Ministério da Saúde, que regulamenta as normas de pesquisa com seres humanos, o estudo foi apreciado pelo Comitê de Ética em Pesquisa da referida instituição e aprovado sob protocolo n ${ }^{0}$ 071/09. 0 acesso aos prontuários e/ou às fichas de atendimento das crianças foi obtido após assinatura do Termo Fiel Depositário pelo diretor da referida instituição.

\section{APRESENTAÇÃO E DISCUSSÃO DE RESULTADOS}

Considera-se importante conhecer algumas variáveis sociodemográficas da população em estudo, para que se possa refletir sobre a relação da realidade da situação vacinal desta.

\section{Caracterização sociodemográfica das crianças expostas ao HIV}

Os dados referentes às características sexo, idade, instituição e município de procedência das crianças em estudo foram apresentadas na Tabela 1.

Tabela 1 - Distribuição do número de crianças, segundo características sociodemográficas. Fortaleza-CE, set-out/2009

\begin{tabular}{|c|c|c|}
\hline Variáveis & $\mathrm{N}$ & $\%$ \\
\hline \multicolumn{3}{|l|}{ Sexo } \\
\hline Masculino & 65 & 52 \\
\hline Feminino & 60 & 48 \\
\hline \multicolumn{3}{|l|}{ Idade - meses } \\
\hline 2 & 65 & 52 \\
\hline $3-5$ & 36 & 28,8 \\
\hline $6-8$ & 11 & 8,8 \\
\hline $9-11$ & 7 & 5,6 \\
\hline $12-15$ & 3 & 2,4 \\
\hline$>15$ & 3 & 2,4 \\
\hline \multicolumn{3}{|l|}{ Instituição } \\
\hline Hospital A & 58 & 46,4 \\
\hline Hospital B & 33 & 26,4 \\
\hline Hospital C & 14 & 11,2 \\
\hline Hospital D & 10 & 8,0 \\
\hline Hospital E & 06 & 4,8 \\
\hline Outros & 04 & 3,2 \\
\hline \multicolumn{3}{|l|}{ Municípios } \\
\hline Fortaleza & 94 & 75,2 \\
\hline Grande Fortaleza & 12 & 9,6 \\
\hline Litoral Leste/Jaguaribe & 08 & 6,4 \\
\hline Sertão Central & 05 & 4,1 \\
\hline Sertão dos Inhamús & 04 & 3,2 \\
\hline Região do Cariri & 02 & 1,6 \\
\hline
\end{tabular}


Consoante a Tabela 1, 65 (52\%) das crianças eram do sexo masculino e 60 (48\%) do feminino, sem diferença de proporção entre as crianças.

Os resultados demonstraram que $65(52 \%)$ crianças iniciaram o esquema vacinal na idade indicada, a partir de dois meses, conforme o PNI, do Ministério da Saúde; entretanto, apresentaram significativa redução da demanda no seguimento, ao avançar a idade. Enfatiza-se que nenhuma criança completou o calendário vacinal, uma vez que as crianças com idade mais avançada estavam com seis anos e dois meses.

Em Fortaleza, destacaram-se três instituições: Hospital A, com 58 (46,4\%) das referências, o que pode se justificar pelo programa de aconselhamento em DST/HIV e 0 atendimento especializado em DST/HIV/Hepatite; o Hospital B, com 33 (26,4\%), no qual se concentraram as referências de ambulatório especializado de HIV/AIDS, Hepatite e Leishmaniose. E, o Hospital C que apresentou $14(11,2 \%)$ referências que dispunha o CRIES do Estado do Ceará. Outras instituições da região metropolitana apresentaram referência em menor proporção.

Sobre a procedência das crianças, Fortaleza obteve percentual significativo com 94 (75,2\%) e, em segundo lugar, $12(9,6 \%)$, provenientes da região Metropolitana, com a representatividade dos respectivos municípios e números de crianças: Caucaia/01, Guaiúba/01, Eusébio/02, Maranguape/ 02, Maracanaú/01, Itaitinga/03 e Pacajús/02. 0 quantitativo de outros municípios do Estado do Ceará foi de 19 (15,3\%).

\section{Situação vacinal das crianças expostas ao HIV}

De acordo com manual do CRIES, as crianças devem iniciar o esquema de vacina injetável contra poliomielite (VIP) aos 60 dias de vida, depois aos 120, 360 dias, com reforço entre quatro e seis anos. ${ }^{9} 0$ esquema vacinal de crianças com suspeita clínica ou diagnóstico definitivo de HIV faz-se conforme o Guia de Tratamento Clínico da Infecção pelo HIV. ${ }^{8}$

A criança que é exposta ao HIV, para desenvolver a imunidade adquirida contra o vírus da poliomielite, deve seguir relação da dose e idade recomendada nas aplicações. Nessas circunstâncias, a mãe infectada precisa ser orientada, por profissionais da saúde, para compreender que o seguimento do calendário deve ser cumprido e, assim, não romper o processo imunológico em desenvolvimento.

A Tabela 2 apresenta a relação entre número de doses da vacina injetável contra pólio (VIP) e a faixa etária da criança, durante 0 atendimento.

Tabela 2 - Número de doses da vacina injetável contra pólio (VIP), segundo a idade da criança no momento da aplicação. Fortaleza-CE, janeiro/2008 a setembro/2009

\begin{tabular}{lll}
\hline Doses - idade (dias) & N & $\%$ \\
\hline $1^{\text {a dose } n=95}$ & 64 & \\
$60-120$ & 18 & 67,3 \\
$121-180$ & 6 & 18,9 \\
$181-240$ & 2 & 6,3 \\
$241-300$ & 5 & 2,1 \\
$>300$ & 95 & 5,3 \\
Subtotal & & 100 \\
$2^{\mathbf{a}}$ dose $n=60$ & 38 & \\
$120-180$ & 11 & 63,3 \\
$181-240$ & 4 & 18,3 \\
$241-300$ & 3 & 6,7 \\
$301-360$ & 4 & 5,0 \\
$>360$ & 60 & 6,7 \\
Subtotal & & 100 \\
$3^{\mathbf{a}}$ dose $n=25$ & 13 & \\
$181-250$ & 2 & 52,0 \\
$251-310$ & 8 & 8,0 \\
$311-370$ & 2 & 32,0 \\
$>371$ & 25 & 8,0 \\
Subtotal & & 100 \\
\hline
\end{tabular}

Pela análise dos registros, $95(76 \%)$ crianças receberam a primeira dose da VIP, embora o quantitativo não tenha mantido o esquema de vacina na $2^{\mathrm{a}}$ e $3^{\mathrm{a}}$ doses subsequentes, que representam $60(48 \%)$ e $25(20 \%)$, respectivamente. Apenas $20 \%$ das crianças completaram o esquema das três doses da VIP.

0 Ministério da Saúde preconiza a idade ideal para aplicação das três doses consecutivas. Entretanto, os dados demonstraram que 64 (67,3\%) crianças estavam na idade recomendada, entre 60 a 120 dias, na primeira dose da VIP. Nas doses posteriores, houve diminuição na pontualidade do calendário, quanto às doses de retorno para as respectivas idades, pois $38(63,3 \%)$ crianças retornaram para segunda dose e 13 (52\%), para a terceira.

A Tabela 3 apresenta registros de aplicações da vacina contra pneumococo (polissacarídica 23 valente e conjugada 7 valente - Pnc7), e a faixa etária das crianças expostas ao HIV. A vacina é mais conhecida como Prevenar $7 \mathrm{~V}$. 
Tabela 3- Número de doses da vacina contra pneumococo, segundo a idade da criança no momento da aplicação. Fortaleza-CE, janeiro/2008 a setembro/2009

\begin{tabular}{lll}
\hline Doses -idade (dias) & $\mathbf{N}$ & $\%$ \\
\hline $\mathbf{1}^{\mathbf{a}}$ dose $\mathrm{n}=105$ & & \\
$60-120$ & 68 & 64,8 \\
$121-180$ & 12 & 11,4 \\
$181-240$ & 11 & 10,5 \\
$241-300$ & 5 & 4,8 \\
$>300$ & 9 & 8,8 \\
Subtotal & 105 & 100 \\
$\mathbf{2}^{\mathbf{a}}$ dose $\mathrm{n}=\mathbf{7 8}$ & & \\
$120-180$ & 34 & 43,5 \\
$181-240$ & 23 & 29,5 \\
$241-300$ & 5 & 6,4 \\
$301-360$ & 8 & 10,2 \\
$>360$ & 8 & 10,2 \\
Subtotal & 78 & 100 \\
$3^{\mathbf{a}}$ dose $\mathrm{n}=33$ & & \\
$190-250$ & 15 & 45,4 \\
$251-310$ & 7 & 21,2 \\
$311-370$ & 5 & 15,1 \\
$371-430$ & 2 & 6,1 \\
$>430$ & 4 & 12,1 \\
Subtotal & 33 & 100 \\
\hline
\end{tabular}

No tocante ao número de doses da vacina contra pneumococo, $105(84 \%)$ crianças receberam a primeira dose; 78 (62,4\%), a segunda e 33 (26,4\%), a terceira dose. Deste modo, alcançou-se um panorama semelhante ao da VIP, entendendo que apenas $26,4 \%$ das crianças completaram o esquema.

Em relação à faixa etária ideal das crianças no momento das aplicações das três doses, 68 (64,8\%) crianças receberam a primeira dose; 34 (43,5\%), a segunda; e $15(45,4 \%)$, a terceira dose. A relação idade adequada e adesão do esquema da Pnc7 observada não seguiu de forma satisfatória, pois os dados apresentaram redução da demanda nas doses subsequentes.

Entre as demais vacinas oferecidas pelo Ministério da Saúde, a primeira dose contra Hepatite $\mathrm{A}$ (HepAdose3), Varicella zoster (VZdose1) e Meningococo tipo C conjugada (MenCconjdose1) foi a mais aplicada em 16,15e 13 crianças, respectivamente. No entanto, o seguimento do calendário sofreu desvio descrescente nas doses subsequentes: HepAdose2 apareceu com duas doses, VZdose2, com nenhuma e a MenCconjdose2 e MenCconjdose3 com sete e duas aplicações, respectivamente.

Os imunobiológicos difteria, tétano e pertussis acelular (DTPa), Haemophilus influenzae tipo b (HIB) e hepatite B (hepB), embora com aplicações inferiores em relação às vacinas destacadas, mantiveram regularidade no seguimento das demais doses recomendadas, ou seja, metade de seis crianças no esquema da DTPa receberam as quatro doses padronizadas; das quatro da HIB, apenas uma recebeu todas as doses, e a única criança vacinada com a HepB obteve todas as doses. Mesmo com pequeno número de aplicações, o desnível na continuidade do esquema vacinal foi menor quando comparadas aos demais imunobiológicos do gráfico.

Gráfico 1- Crianças vacinadas em relação aos imunobiológicos aplicados no período de janeiro de 2008 a setembro de 2009 no CRIE de Fortaleza- CE.

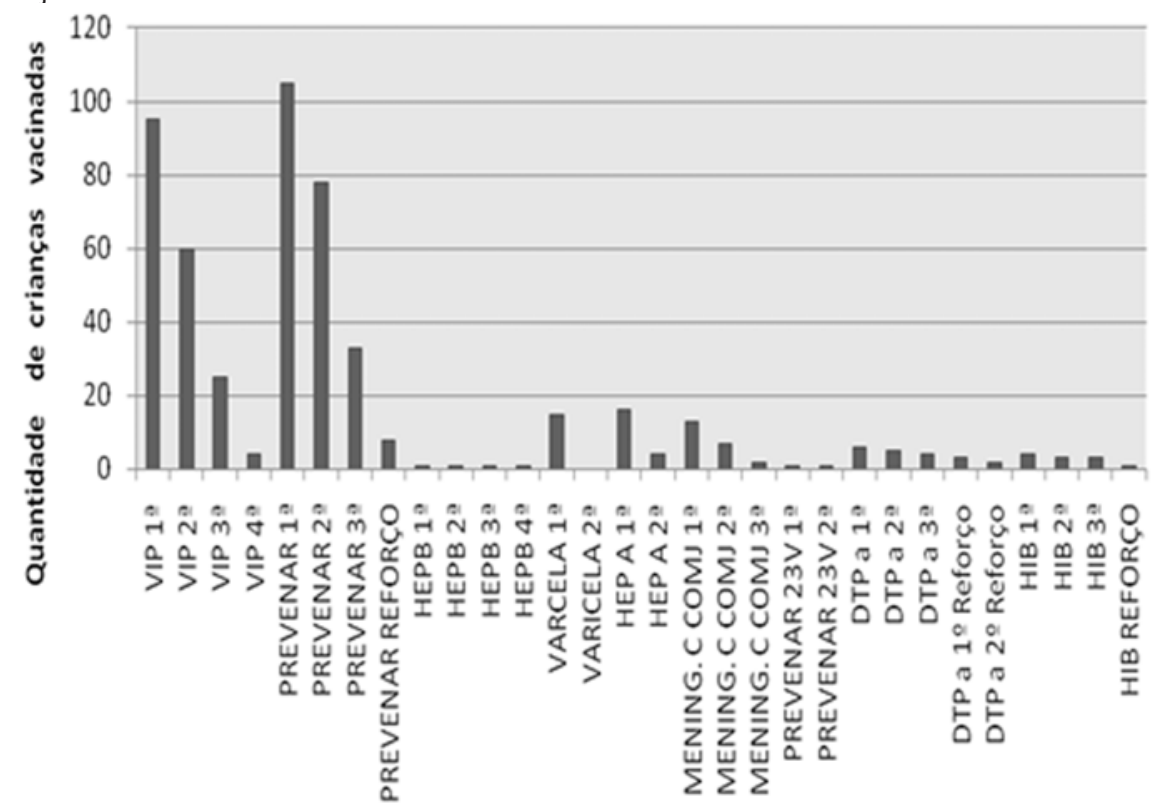

Imunológico 


\section{DISCUSSÃO}

Em geral, as crianças iniciam a imunização na idade recomendada, porém sem adesão às doses subsequentes do esquema. Enfatiza-se que de acordo com o PNI do Brasil, finalizase o calendário vacinal entre 14 e 16 anos de idade. Todavia, com base nos dados, a maior idade obtida foi de 6 anos e 2 meses, o que demonstra que nenhuma criança completou 0 calendário, mesmo iniciando a imunização no período adequado.

É importante ressaltar que a maioria das crianças residia na capital do Estado, o que favorece a acessibilidade ao CRIES, visto que a descontinuidade ao esquema de vacina é significativa.

0 fator acessibilidade foi cogitado como possível problema impeditivo de mães de crianças expostas frequentarem o CRIES, no período de aprazamento; porém, conforme dados demográficos, apenas $24,8 \%$ da amostra não residiam no município locus do estudo, ou seja, a maioria das crianças expostas (75,2\%) residia em Fortaleza e 9,6\% na Grande Fortaleza, que representa os municípios de São Gonçalo do Amarante, Caucaia, Maracanaú, Guaiúba, Maranguape, Pacatuba, Horizonte, Pagajus, Cascavel, Chorozinho, Aquiraz, Pindoretama, Itaitinga e Eusébio. Assim, o acesso ao CRIES não se caracteriza como fator impeditivo para a descontinuidade do esquema de vacina especial.

Apenas 20\% das crianças completaram as três doses da VIP. Em conformidade com as normas do PNI, como resposta imunológica após aplicação de duas doses, a VIP confere proteção sérica a $95 \%$ dos receptores, e, nas três doses, promovem-se $99 \%$ a $100 \%$, com altos títulos de anticorpos. A imunidade é de longa duração, mas recomendam-se mais duas doses de reforço. ${ }^{7}$

Nesse contexto, a ausência de uma das doses associada à ineficiência do sistema imune presente no organismo da pessoa com HIV imunodeprimida, possivelmente, repercute na ação de imunização da VIP, comprometendo o processo de imunidade que se iniciou, pois conforme o Manual dos CRIES, à medida que se intensifica a imunodepressão, aumenta-se 0 risco da aplicação de vacinas de agentes vivos, bem como a possibilidade de resposta imunológica insuficiente ou inadequada. ${ }^{7}$

Devem-se enfatizar os riscos relacionados aos familiares da criança exposta ao HIV, considerando que pelos menos os pais são portadores do HIV e precisam também estar imunologicamente seguros através da vacinação especial, não somente para prevenir as infecções oportunistas, mas porque indivíduos que convivem com pessoa infectada pelo HIV devem receber vacina inativada contra a poliomielite (VIP), quando indicado, vacina contra a varicela, se suscetíveis, e vacina inativada contra a influenza. ${ }^{8}$

Em relação a Pnc7, há evidências de que há diminuição do número de portadores do pneumococo em nasofaringe dos vacinados. Resposta sorológica adequada, após esquema completo da Pnc7, tem sido observada em crianças com HIV/ AIDS, doença de Hodgkin e anemia falciforme.?

Embora o quantitativo das doses de Pnc7 tenha sido superior às demais vacinas até mesmo da VIP, a descontinuidade do aprazamento foi visível. Além disso, a relação dose e idade recomendada não foi seguida, o que poderia comprometer 0 processo de imunidade quanto à Pnc7.

Assim, a baixa adesão e o descumprimento da relação dose e idade do calendário especial se apresenta semelhante ao da VIP, evidenciando que a minimização do número de doses não se limitou apenas a uma vacina e que os possíveis fatores da descontinuidade ao esquema especial precisam ser investigados em estudos futuros, embora algumas causas tenham sido apontadas no decorrer desta pesquisa.

Essa situação é semelhante à das doses observadas no gráfico, se apresentando como mais um demonstrativo da dificuldade de adesão ao seguimento do calendário, que podem estar relacionadas à disponibilidade dos imunobiológicos, à credibilidade e ao entendimento da mãe ao processo preventivo das vacinas e às orientações de profissionais, com a finalidade de estimular e motivar a mãe infectada a aderir ao calendário vacinal.

Diante da problemática, provavelmente, as mães de crianças expostas supõem que o filho não desenvolverá a infecção e, assim, negligenciam as doses do esquema de vacinação especial. Contudo, as crianças com exposição neonatal ao HIV recebem as mesmas recomendações das diagnosticadas como infectadas, até a definição de não infectadas, pois mesmo que não se revelem com o vírus, convivem com pessoas HIV. ${ }^{11}$

Ao investigar o cuidado da mãe infectada na prevenção da transmissão vertical, um estudo comprovou irregularidades em relação ao cuidado dispensado às crianças, sob risco da aquisição do HIV. As dificuldades de adesão advêm muito mais da carência de conhecimento das mães acerca da prevenção e da omissão dos profissionais e dos serviços de saúde do que de limitações pessoais no cuidado ao filho, ${ }^{12}$ o que pode acarretar em implicações também para o acompanhamento imunológico especial.

Em comparação a estudo sobre adesão à terapia antirretroviral, ao contrário de outras doenças crônicas, a criança com HIV, na maioria, vive em situação de pobreza crônica, recursos limitados, com discriminação, desagregação familiar, cuidadores dependentes químicos, estressores dos grandes centros urbanos, e, dessa forma, repercute na ausência de motivação e continuidade aos tratamentos recomendados. ${ }^{13}$

Nessa perspectiva, a atitude da família/cuidador é fundamental para a aderência pediátrica, pois lactentes e crianças menores dependem, quase que inteiramente, de cuidador, na administração de medicamentos, bem como na condução de numerosos cuidados à criança exposta ou com $H I V,{ }^{13}$ dentre os quais o seguimento adequado da imunização especial. 
Promover e manter medidas preventivas e tratamento adequado das doenças ainda é uma das principais estratégias de diminuição das infecções oportunistas que ainda acometem esse público, apesar do progresso da terapia antirretroviral de melhoria de resposta imunológica das crianças com HIV. ${ }^{14} \mathrm{Além}$ disso, é essencial o suporte da família, mediante orientaç̃̃es educativas dos profissionais, na adesão ao tratamento antirretroviral e também a toda e qualquer terapêutica para diminuir a morbimortalidade, bem como melhorar a qualidade de vida das crianças.

A atitude da criança portadora do HIV, ao lidar com a doença e o tratamento, é reflexo do cuidado da mãe e dos familiares em relação ao HIV. A mãe alheia ao próprio cuidado como portadora do HIV deve entender que ao negligenciar o tratamento influencia negativamente no processo de enfrentamento e compromete o cuidado ao filho portador. As mães e os familiares precisam ser conscientizados acerca da necessidade de apoio, segurança e seguimento da terapêutica relacionada ao HIV, além da prevenção de doenças oportunistas no cenário familiar. ${ }^{15}$

Os profissionais de saúde, em todos os níveis de atenção, são corresponsáveis pelo cuidado desempenhado pelos familiares à criança, por meio de estratégias que promovam a conscientização e compreensão acerca da infecção pelo HIV e suas complicações, considerando as recomendações do Ministério da Saúde de que crianças, adolescentes e adultos expostos ou infectados pelo HIV, na ausência de alterações imunológicas e clínicas com indicação de imunodeficiência, devem receber todas as vacinas de rotina do calendário nacional, assim como as vacinas especiais.

Na perspectiva de melhor conscientização da gestão institucional e dos profissionais dos centros de referência, enfatiza-se a necessidade do funcionamento eficaz do serviço especializado e de referência com base na qualidade e não apenas como preservação de rótulo. ${ }^{12}$

\section{CONCLUSÃO}

A prática do esquema vacinal de crianças com suspeita clínica ou diagnóstica definitiva para HIV continua sendo um grande desafio aos profissionais de saúde que prestam assistência às crianças, em unidade hospitalar e/ou centro de saúde.

Conforme resultados, a maioria das crianças iniciou o esquema vacinal com dois meses de idade, ou seja, no período preconizado; entretanto, apresentaram significativa redução na demanda com o avançar da idade. Nessa realidade, deparamse crianças e o respectivo cartão de vacina, sem a administração de imunobiológicos, apresentando-se de forma incompleta ou atrasada.

É perceptível que, das vacinas do esquema especial oferecidas pelo CRIES, a VIP e a Pnc7 foram as que apresentaram o maior quantitativo de doses, todavia a não adesão ao seguimento delas foi evidente, o que, possivelmente, repercute em alteração imunológica inadequada. Ressaltase que no período da coleta de dados, não houve indisponibilidade da VIP na instituição, apontando que a não adesão deveria estar relacionada a outros fatores, os quais não foram objeto de estudo desta pesquisa.

Assim, esse demonstrativo do seguimento do esquema especial constitui-se em um alerta aos gestores e profissionais de saúde, compreendendo que as mães infectadas e as crianças expostas ao HIV integram um grupo de risco para doenças preveníveis, as quais, por sua vez, tornam-se oportunistas pelas condições de saúde impostas pela infecção.

Nesse caso, a imunização das crianças expostas ao HIV deveria ser acompanhada regularmente pelos gestores e profissionais da saúde inseridos no contexto da saúde pública, particularmente de políticas públicas com vistas a avaliar os programas de prevenção de doenças, especialmente os relacionados às doenças oportunistas decorrentes da infecção pelo HIV.

Considerando as consequências do rompimento imunológico que vem se desenvolvendo junto às crianças expostas ao HIV, a baixa adesão percebida, seus fatores determinantes, bem como suas implicações para o processo saúde-doença precisam ser investigados com mais profundidade, o que se apresentou como uma das limitações deste estudo.

Uma das dificuldades vivenciadas para a realização do estudo que merece destaque foi a deficiência de alguns registros nos prontuários das crianças do CRIES em estudo, como também a ausência de informações estatísticas de atendimentos por faixa etária e dados sociodemográficos da população assistida.

Com base na evidência de baixa adesão ao calendário vacinal, urge a necessidade do desenvolvimento de ações de educação em saúde com os profissionais de saúde que atuem diretamente com essa clientela para que estes, consequentemente, ampliem as temáticas relacionadas à exposição ao HIV e incluam a imunização especial como uma das estratégias para auxiliar na prevenção de doenças e comorbidades.

Nesse contexto, a Enfermagem possui papel importante no processo saúde-doença da criança e família, o que torna imprescindível a realização de um trabalho educativo a respeito da significância do calendário vacinal, dos imunobiológicos, das normas do PNI para os profissionais de saúde, ademais a conscientização da população. 


\section{REFERÊNCIAS}

1 Ministério da Saúde (BR). Guia de tratamento clínico da infecção pelo HIV em pediatria. $3^{\mathrm{a}}$ ed. Brasília: Ministério da Saúde; 2007.

2 Ministério da Saúde (BR). Programa Nacional DST/AIDS. Bol Epidemiol AIDST. [on-line] 2008 jan/jun; [citado 2009 dez 14]; 5(1): [aprox. 64 telas]. Disponível em: http://portal.saude.gov.br/saude.

3 Ministério da Saúde (BR). Direitos sexuais e direitos reprodutivos: uma prioridade do governo. Brasília: Ministério da Saúde; 2005.

4 Stefani M, Araújo BF, Rocha NMP. Transmissão Vertical do HIV em população de baixa renda do sul do Brasil. DST J Bras Doenças Sex Transm. 2004; 16(2):33-9.

5 Lima GS, Braga TDA, Meneses JA, coordenadores. Neonatologia: Instituto Materno-Infantil de Pernambuco (IMIP). Recife: Guanabara Koogan; 2004.

6 Ministério da Saúde (BR). Recomendações para terapia antirretroviral em crianças e adolescentes infectados pelo HIV [Internet]. Brasília: DF; 2009. Disponível em: http://www.aids.gov.br/data/documents/storedDocuments/ \%7BB8EF5DAF-23AE-4891-AD36-1903553A3174\%7D/\%7B887610527FCB-41BD-B4D8-0A1EF9E0BBAA\%7D/con-crianca\%2001.pdf

7 Fundação Nacional de Saúde (BR). Manual dos Centros de Referências de Imunobiológicos Especiais - CRIES. $3^{\mathrm{a}}$ ed. Brasilia: Ministério da Saúde; 2006.

8 Ministério da Saúde (BR). Doenças e condições que podem ter necessidades de vacinas especiais disponíveis gratuitamente nos CRIES: orientação ao público. Brasilia: Ministério da Saúde; 2006.

9 Ministério da Saúde (BR). Vacinas complementares fornecidas pelos Centros de Referências de Imunobiológicos Especiais (CRIES): orientação à classe médica: relação das vacinas complementares indicadas por patologia. Brasília: Ministério da Saúde; 2007.

10 Carvalho ALA, Sousa FGM, Santos MH. Situação vacinal de estudantes de enfermagem e adesão ao programa de imunização de adultos. Online Braz J Nurs [periódico na Internet]. 2006; [citado 2010 julho 25]; 5(3). Disponivel em: http://www.objnursing.uff.br/index.php/nursing/article/view/ $428 / 101$

11 Sztajnbok DCN. Imunização em crianças e adolescentes infectados pelo HIV. Rev Hosp Univ Pedro Ernesto. 2007 jan/jun; 6(3):56-65.

12 Barroso LMM, Galvão MTG, Cavalcante RM, Freitas JG. Cuidado materno aos filhos nascidos expostos ao HIV. Rev Rene. 2009 out/dez; 10(4):15564.

13 Simoni JM, Montgomery A, Martin E, New M, Demas PA, Rana S. Adherence to Antiretroviral Therapy for Pediatric HIV Infection: A Qualitative Systematic Review with Recommendations for Research and Clinical Management. Pediatrics. 2007 jun; 119(6):e1371-83.

14 Mofenson LM, Brady MT, Danner SP, Dominguez KL, Hazra R, Handelsman E, Havens $P$ et al. Guidelines for the Prevention and Treatment of Opportunistic Infections among HIV-exposed and HIV-infected children: recommendations from CDC, the National Institutes of Health, the HIV Medicine Association of the Infectious Diseases Society of America, the Pediatric Infectious Diseases Society, and the American Academy of Pediatrics. MMWR Recomm Rep. 2009 sep 4; 58(RR-11):1-166.
15 Paula CC, Crossetti MGO. Existencialidade da criança com AIDS: Perspectivas para o cuidado de enfermagem. Esc Anna Nery Rev Enferm. 2008 mar; 12(1):30-8.
Recebido em 25/08/2010

Reapresentado em 17/02/2011 Aprovado em 12/05/2011 